\title{
Donepezil can improve daily activities and promote rehabilitation for severe Alzheimer s patients in long-term care health facilities
}

\author{
Kenichi Meguro ${ }^{*}$, Yoshitaka Ouchi, Kyoko Akanuma, Mitsue Meguro and Mari Kasai
}

\begin{abstract}
Background: Cholinesterase inhibitors can delay the progression of Alzheimer s disease (AD). Several clinical trials of the drug in moderate to severe AD have consistently reported clinically positive effects. A combining effect with psychosocial intervention was reported in mild to moderate AD patients. Since a therapeutic approach or rehabilitation combined with cholinesterase inhibitors for severe AD patients remains controversial, we performed a prospective intervention for patients in Long-Term Care Health Facilities (LTCHF).

Methods: Two LTCHFs (N1, N2) were enrolled. N1 is a 126-bed facility that does not treat with donepezil but rather with psychosocial intervention (reality orientation and reminiscence). N2 is a 150-bed facility with a 50-bed special dementia unit, in which the physician can prescribe donepezil. On top of the similar psychosocial intervention, rehabilitation is performed in N2. Thirty-two severe AD patients (MMSE <6) in N1 and N2 (16 vs. 16) were compared for the effect of donepezil (10 mg/d for 3 months) with or without psychosocial intervention ( $n=8 \mathrm{vs} .8$ for each facility). The Vitality Index was used to assess daily activities and the introduction of rehabilitation.

Results: The response ratio (MMSE 3+) of donepezil was 37.5\% in N2. The combination of donepezil with the psychosocial intervention improved the Vitality Index total score, and Communication, Eating, and Rehabilitation subscores (Wilcoxon, $p=0.016,0.038,0.023$, and 0.011 , respectively). Most of them were smoothly introduced to rehabilitation, and the proportion of accidental falls decreased. Psychosocial intervention in N1 without the drug only improved the total score (Wilcoxon, $\mathrm{p}=0.046$ ).

Conclusions: A combined therapeutic approach of donepezil and psychosocial intervention can have a positive effect, even for severe patients through the introduction of rehabilitation and decreasing accidental falls. However, these findings require replication in a larger cohort.
\end{abstract}

Keywords: Alzheimer s disease, Donepezil, Cholinesterase inhibitors, Life expectancy, Nursing home

\section{Background}

The number of Alzheimers disease (AD) patients continues to increase worldwide and thus the establishment of an appropriate therapeutic approach including drug, psychosocial intervention, and rehabilitation is necessary. For drug treatment, cholinesterase inhibitors (ChEIs), such as donepezil, have clinically positive effects, leading to improvements in cognitive function for a certain duration and delaying the progression of the disease [1]. The drug can delay the deterioration of daily function [2], thus decreasing the caregivers burden [3]. Indeed, the severity of

\footnotetext{
* Correspondence: k-meg@umin.ac.jp

Division of Geriatric Behavioral Neurology, CYRIC, Tohoku University, 2-1, Seiryo-machi, Aoba-ku, Sendai, Miyagi 980-8575, Japan
}

the daily functional disability is the most critical factor in keeping $\mathrm{AD}$ patients living in their homes prior to placement in nursing homes [4].

Most AD patients residing in nursing homes have severe stage of disease characterized by a profound cognitive loss as well as deterioration in functional abilities [5]. Thus, the treatment of severe AD is expected to improve or stabilize such symptoms [6,7]. Several clinical trials of ChEIs in moderate to severe $\mathrm{AD}$ [8-11] have consistently reported such effects. Jelic et al. [12] studied the efficacy of donepezil for severe AD patients in 248 nursing homes, and demonstrated that donepezil treatment showed stabilization or improvement across multiple outcome measures. 
Since ChEIs are not curative drugs, the addition of psychosocial interventions are also considered in order to optimize the function of patients. The reminiscence approach [13] seeks to facilitate the recall of past experiences and thereby improve well-being or quality of life (QOL). Relatively well preserved remote memory in AD [14] can provide a neurological basis to support this approach. The reality orientation (RO) technique $[13,15]$ is also used, which stimulates time and place orientation, usually by providing information regarding orientation. It is usually used in combination with the reminiscence approach $[16,17]$.

We have previously reported a positive effect of combined donepezil and psychosocial intervention, including the $\mathrm{RO}$ with the reminiscence approach, on the patients QOL [18]. However, such positive effects of the drug are not always recognized by the patients and caregivers; thus, leading them to discontinue the drug treatment easily [19]. Umegaki et al. [20] surveyed the persistence of donepezil therapy and found that only $50 \%$ of AD patients remained on treatment after one year. The main reason for drug withdrawal was the perceived ineffectiveness of the drugs by the patients and caregivers, which may contrast with their unrealistic expectations, such as improving memory function.

Although benefit of psychosocial intervention such as $\mathrm{RO}$ and physical reactivation program in combination with donepezil for mild to moderate $\mathrm{AD}$ has been reported [21], no such studies have been performed for patients with severe stage of disease. Such a combined therapeutic approach especially for severe $\mathrm{AD}$ patients would be better performed at the institutes, since caregivers are also getting old and even suffering from mild cognitive impairment in Japan, and high-quality psychosocial intervention is not frequently possible at home.

According to the Long-Term Care Insurance, three kinds of institutes are available in Japan, i.e., the Long-Term Care Health Facilities (LTCHF), Special Elderly Nursing Homes, and Group Homes. The situations of drug treatment are different: patients living in the latter two institutes are able to consult doctors at other clinics to receive prescriptions. However, after placement in the LTCHF, by law the patients can only take medicines provided by the institute, and unfortunately expensive drugs, such as ChEIs, are not always provided. Managers of the LTCHFs decide independently whether or not the ChEIs are provided for economic reasons. We believe that this is a second reason for drug withdrawal [19].

However, we actually experienced patients with severe $\mathrm{AD}$ who experienced a positive effect by the combination of donepezil and psychosocial intervention. It may be due to the introduction of rehabilitation that was reported to improve ADL. The aim of the study is to investigate whether ChEIs together with psychosocial interventions have clinically positive effects for patients with severe AD. We hypothesized that even for LTCHFreplacement patients with severe $\mathrm{AD}$, ChEIs together with psychosocial interventions have such effects. This effect included improving daily activities and promoting rehabilitation, as well as decreasing the incidence of accidental fall. Data was collected prospectively from the two types of LTHCFs.

\section{Methods}

LTCHF

Two LTCHFs (N1, N2), which are located in Miyagi Prefecture, Northern Japan, were enrolled. They are run by the same company and have a close relationship with our research group. N1 is a 126-bed facility without donepezil but psychosocial intervention (reality orientation and reminiscence) is performed. N2 is a 150-bed facility with a 50-bed special dementia unit. After the approval of donepezil (see below), physicians became able to prescribe donepezil at that site. In addition to the similar psychosocial intervention, rehabilitation is performed in N2.

\section{Patients}

Thirty-two severe patients with AD (16 in N1 and 16 in N2) were studied. The inclusion criteria were: 1 ) to meet the diagnostic criteria for probable $\mathrm{AD}$ according to the NINCDS-ADRDA [22] with reference to the CT or MRI images; 2) the scores on the Mini-Mental State Examination (MMSE) [23] were less than 6;3) more than three months had passed since placement in N1 or N2; and 4) they were all able to walk with or without canes and eat with the help of the staff.

The exclusion criteria were 1) significant neurological signs, such as hemiparesis or swallowing disturbance; 2 ) the presence of aphasia that prevented them from verbal communication with the staff; 3) severe behavioral symptoms, such as delusion or delirium and wandering, that prevented them from joining psychosocial intervention or rehabilitation; 4) history of stroke, cerebral contusion, or other systematic disorders that could affect central nervous system function, i.e., hypothyroidism or decreases in vitamin $\mathrm{B}_{1}, \mathrm{~B}_{6}, \mathrm{~B}_{12}$.

Informed consent was received from the family members of all patients analyzed. This study was approved by the Medical Ethics Committee of Tohoku University and those of LTHCF N1 and N2.

\section{Psychosocial intervention in N1 and N2}

The RO and reminiscence approaches are routinely performed as a group work in N1 and N2. Sixteen patients in $\mathrm{N} 1$ and $\mathrm{N} 2$ were randomly divided into two arms, i.e., those who received psychosocial intervention $(n=8$, each for $\mathrm{N} 1$ and $\mathrm{N} 2$ ) and those who received normal care 
( $\mathrm{n}=8$, each for N1 and N2). Rehabilitation for walking was also performed by a physical therapist in $\mathrm{N} 2$.

The characteristics of N1 and N2 facilities and the information on the patients demographic and clinical characteristics the patients studied are shown in Table 1. Baseline characteristics are the same through the groups.

\section{The use of Donepezil in N2}

After explanation of the study protocol, a manager at N2 agreed to employ the $3 \mathrm{mg}, 5 \mathrm{mg}$, and $10 \mathrm{mg}$ tablets of donepezil. After confirming the absence of side effects in response to $3 \mathrm{mg} /$ day for 1 or 2 weeks, $10 \mathrm{mg} /$ day of donepezil was administered for 16 patients in N2.

\section{Outcomes}

To address the hypothesis described above, the three outcomes below were measured pre- and post-interventions. Post-intervention assessments were performed after a period of 3 months. During this three-month period, the drugs were not changed.

\section{Vitality index}

The Vitality Index [24] was used to assess daily activities and the introduction of rehabilitation. This index is established and validated by Toba et al., comprised of five items, with each of them being assessed according to 3 ratings, as follows: 1 ) Waking pattern (2: organized pattern of waking, 1: requires a caregiver $\mathrm{s}$ aid occasionally, 0: Never wakes voluntarily), 2) Communication (2: vocalizes reciprocal exchanges at will, 1: responsive to verbal stimulation, 0: no cognitive response), 3) Feeding (2: motivated to eat, 1: passive, but eats with encouragement, 0: indifferent to eating), 4) On and off toilet (2: independent or never fails to express micturition desire, 1 : does not express micturition desire consistently, 0 :

Table 1 Donepezil administration for AD patients in LTCHF (n = 32) +/- Psychosocial Intervention

\begin{tabular}{llll}
\hline & & LTCHF-N1 & LTCHF-N2 \\
\hline \multirow{2}{*}{$\begin{array}{l}\text { Dementia ward } \\
\text { Donepezil }\end{array}$} & 126 beds & 150 beds \\
Subjects & & None & 50 beds \\
Usual care & $n$ & $(16$ patients) & $(16$ patients $)$ \\
& M/F & 8 & 8 \\
& MMSE Mean (SD) & $3.6(1.1)$ & $3.5(0.9)$ \\
Psychosocial & Contents & RO + & RO + Reminiscence, \\
intervention & & Reminiscence & Rehabilitation \\
& $n$ & 8 & 8 \\
& $\mathrm{n}$ & $2 / 6$ & $2 / 6$ \\
& M/F & $3.6(1.2)$ & $3.6(1.4)$ \\
\hline
\end{tabular}

$\mathrm{AD}=$ Alzheimer $s$ disease, $\mathrm{LTCHF}=$ Long-Term Care Health Facility, RO = Reality Orientation, $\mathrm{M}=$ male, $\mathrm{F}=$ female, $\mathrm{MMSE}=$ Mini Mental Sate Examination. indifferent to voiding), 5) Rehabilitation and other activities (2: motivated to be rehabilitated or to be involved in other activities, 1: passive but tries with encouragement, 0 : refuses or indifferent). The staffs at the two LTCHFs receive joint uniform training in usage of the scale.

\section{MMSE}

The MMSE was used to assess general cognitive function.

\section{Accidental falls in N2}

The records of risk management in N2 before and after donepezil administration were analyzed retrospectively.

\section{Analyses}

Analysis 1 (N1): effect of psychosocial intervention without donepezil

Sixteen patients in N1 did not receive donepezil. Eight patients undergoing normal care and 8 patients who underwent the $\mathrm{RO}$ and reminiscence approaches were compared to reveal the effect of psychosocial intervention in the absence of donepezil.

\section{Analysis 2 (N2): effect of psychosocial intervention combined with donepezil}

Sixteen patients in N2 received donepezil. Eight patients undergoing normal care and 8 patients who underwent the RO and reminiscence approaches were compared to reveal the effect of psychosocial intervention combined with donepezil.

\section{Analysis 3: accidental falls (N2)}

The records of risk management in N2 before and after donepezil administration were analyzed retrospectively. The accidents were categorized to Fall, Bruise, Drug, Swallow, Bed, and Loss. Fall herein means fall down on the floor during walking, bruise shows any kind of bruise on their bodies, drug indicates mal-taking medicines provided for other patients, swallow means swallowing something by mistake, bed shows any kind of troubles around the beds, and loss indicates going out of the institutes by mistake.

Non-parametric Wilcoxon matched-pairs signed-rank tests were performed to reveal pre and post differences for Analyses 1 and 2. The Chi-square test was used for Analysis 3. The significance levels were all set at 0.05 . The IBM SPSS Statistics 22.0 was used.

\section{Results}

All 32 patients completed the intervention. None of the patients exhibited acute diseases, such as pneumonia or fever that would warrant excluding the patients from the study protocol. 


\section{Analysis 1 (N1): effect of psychosocial intervention without donepezil}

Figure 1 illustrates the changes of the Vitality Index total scores for psychosocial intervention in N1. The normal care group showed no significant changes; however, the psychosocial intervention group exhibited improvement in the total scores (Wilcoxon, $\mathrm{p}=0.046$ ). No significant differences were noted for the Vitality Index subscores.

\section{Analysis 2 (N2): effect of psychosocial intervention combined with donepezil}

Figure 2 illustrates the changes of the Vitality Index total scores for psychosocial intervention combined with donepezil in N2. Similar to N1, the normal care group showed no significant changes; however, the psychosocial intervention group exhibited improvement in the total scores (Wilcoxon, $\mathrm{p}=0.016$ ). The Vitality Index subscores of Communication $(p=0.038)$, Eating $(p=0.023)$, and Rehabilitation ( $\mathrm{p}=0.011)$ were also improved.

Table 2 notes the changes of MMSE, observed behavior and cognition in 16 patients in N2. The IDs \#1 through \#8 received donepezil and normal care only, and \#9 through 16 received donepezil and psychosocial intervention. Since six patients (IDs\#9-11 and \#14-16) showed an increase of 3 or more MMSE points during the 3 months follow-up (MMSE 3+ improvement), the response ratio of donepezil was calculated as $37.5 \%$ (6/ 16). Eight patients (IDs \#9 through 16) were introduced smoothly to rehabilitation and one patient (ID \#9) was discharged from N2 and returned to her home. The case report will be presented below for better understanding of these results.

\section{Case report}

The patient (\#ID 9 in Table 2) was an 89-year-old woman. She got married when she was 24 years old. Her husband was killed in World War II when she was 25 y. Thereafter, she obtained a hairdresser s license and ran a barber shop with her nephew. When she was 84 years old, she had difficulty in financial management and closed her barber shop. When she was 86 years old, she had a difficulty in maintaining hygiene in the house. Since her room had become verminous, a public health nurse forced her to be placed in N2. Her MMSE score was 7. After living in $\mathrm{N} 2$, she had nothing to do and just spent her time watching TV.

Taking into consideration her past life history, we prepared hair mannequins for her rehabilitation. A session was made up of occupational therapy of hair dressing using hair mannequins, together with reality orientation and the reminiscence approach. We performed one hour per day per week for 3 months. At the beginning, she told us that she had never seen hair mannequins when they were initially presented to her. After administration of donepezil $5 \mathrm{mg} /$ day combined with the rehabilitation, her attention was stimulated. After 2 months, she recalled that she had been a hairdresser and indulged in reminiscence about her past life. She claimed that other patients were lacking in sanitation. Although her MMSE score was not increased to 8 , her Vitality Index increased dramatically from 1 to 6 , with increases for all subscores. A physical therapist reported that she had become active and had more attention for the rehabilitation program of standing and gait. After three months, she was finally discharged from N2 and returned to her home and started to live together with her nephew.

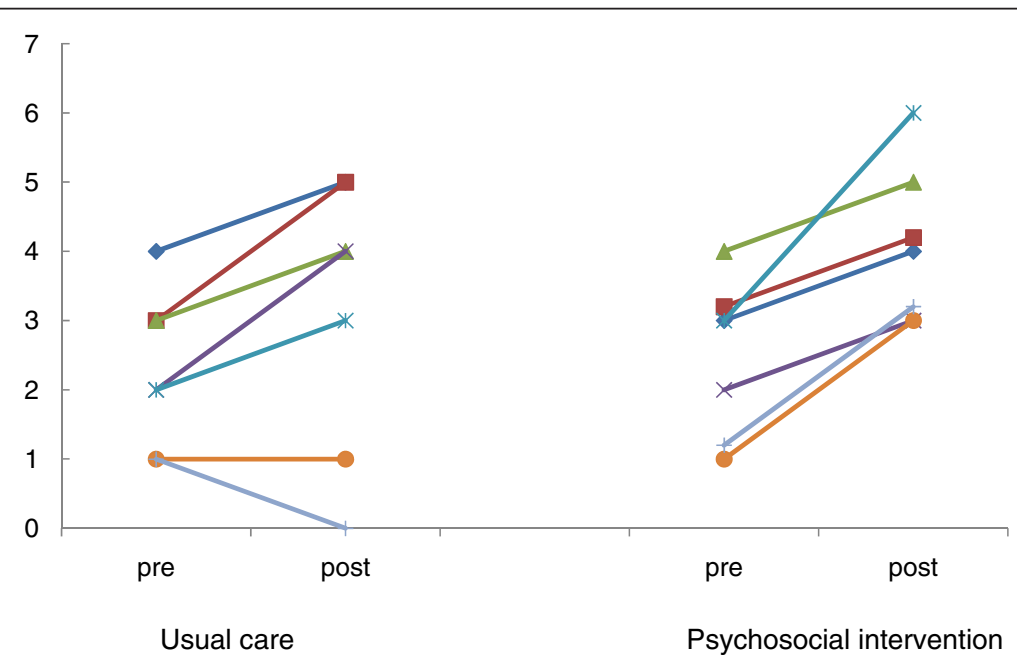

Figure 1 The normal care group showed no significant changes; however, the psychosocial intervention group exhibited improvement in the total scores (Wilcoxon, $\mathbf{p}<\mathbf{0 . 0 5}$ ). No significant differences were noted for the Vitality Index subscores. Vitality Index changes for psychosocial intervention in LTCHF-N1. 


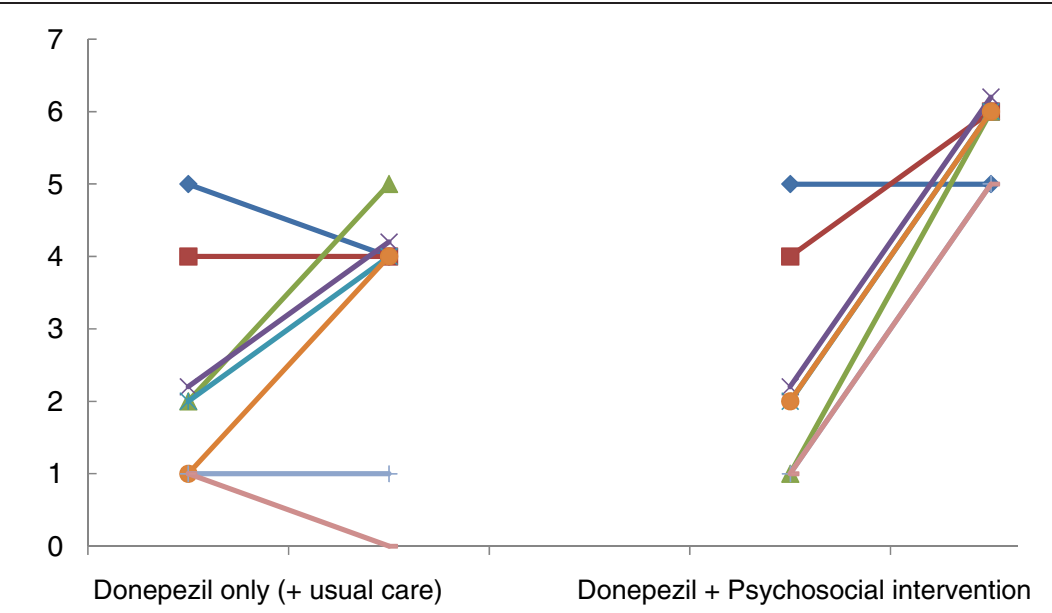

Figure 2 Similar to N1, the normal care group showed no significant changes; however, the psychosocial intervention group exhibited improvement in the total scores (Wilcoxon, $\mathbf{p}<\mathbf{0 . 0 5}$ ). The Vitality Index subscores of Communication, Eating, and Rehabilitation were also improved (Wilcoxon, $\mathrm{p}<0.05$ ). Vitality Index changes for donepezil with or without psychosocial intervention in LTCHF-N2.

\section{Analysis 3: accidental falls in $\mathrm{N} 2$}

Table 3 notes the records of risk management in N2 before (6 months, April to September 201X) and after (October 201X $\sim$ March 201X + 1) donepezil administration. The exact year was masked by the N2 staff. The number of accidental Fall decreased significantly after donepezil administration (chi-square test at 0.05). The staff reported that the patients who had received donepezil had

Table 2 Donepezil administration to AD patients in LTCHF-N2

\begin{tabular}{|c|c|c|c|c|c|c|}
\hline \multirow[t]{2}{*}{ ID } & \multirow{2}{*}{$\begin{array}{l}\text { MMSE } \\
\text { pre }\end{array}$} & \multirow{2}{*}{$\begin{array}{l}\text { Psychosocial } \\
\text { Intervention }\end{array}$} & \multicolumn{2}{|l|}{ Changes } & \multirow{2}{*}{\multicolumn{2}{|c|}{$\begin{array}{l}\text { MMSE } \\
\text { post }\end{array}$}} \\
\hline & & & Behavior & Cognition & & \\
\hline 1 & 5 & \multirow[t]{8}{*}{ None } & \multirow{4}{*}{$\begin{array}{l}\text { Decreased } \\
\text { refusal of } \\
\text { being cared }\end{array}$} & \multirow{6}{*}{$\begin{array}{l}\text { Conversation } \\
\text { with the staff }\end{array}$} & 5 & \\
\hline 2 & 5 & & & & 4 & \\
\hline 3 & 4 & & & & 4 & \\
\hline 4 & 4 & & & & 3 & \\
\hline 5 & 3 & & \multirow[t]{4}{*}{ No changes } & & 3 & \\
\hline 6 & 3 & & & & 2 & \\
\hline 7 & 3 & & & \multirow[t]{2}{*}{ No changes } & 2 & \\
\hline 8 & 2 & & & & 5 & \\
\hline 9 & 5 & \multirow[t]{8}{*}{ Done } & \multirow{8}{*}{$\begin{array}{l}\text { Promote } \\
\text { rehabilitation }\end{array}$} & \multirow{8}{*}{$\begin{array}{l}\text { Conversation } \\
\text { with the staff }\end{array}$} & 9 & * \\
\hline 10 & 5 & & & & 8 & * \\
\hline 11 & 4 & & & & 7 & * \\
\hline 12 & 4 & & & & 5 & \\
\hline 13 & 4 & & & & 3 & \\
\hline 14 & 3 & & & & 6 & * \\
\hline 15 & 2 & & & & 6 & * \\
\hline 16 & 2 & & & & 5 & * \\
\hline
\end{tabular}

$\mathrm{AD}=$ Alzheimer $\mathbf{s}$ disease, $\mathrm{LTCHF}=$ Long-Term Care Health Facility, MMSE $=$ Mini-Mental State Examination.

*donepezil responders as shown by MMSE $3+$. developed more attention to their environment and were more cautious in preventing falls.

\section{Discussion and consclusions}

As described in the introduction, several clinical trials of ChEIs in moderate to severe AD have consistently reported clinically positive effects. A combining effect with psychosocial intervention was reported in mild to moderate $\mathrm{AD}$ patients. We herein performed a combining approach for severe AD patients in LTCJFs, and found that a combined therapeutic approach of donepezil and psychosocial intervention can have a positive effect, through the introduction of rehabilitation and decreasing accidental falls.

\section{Effect of psychosocial intervention}

The results in Analysis 1 (N1) demonstrated that psychosocial intervention, including the $\mathrm{RO}$ and reminiscence approach, was effective in the absence of donepezil administration. However, the effect was considered to be weaker than that achieved in combination with the drug (Analysis 2 (N2)), since no significant differences were noted in the subscores.

Table 3 Risk management before/after donepezil deployment in N2

\begin{tabular}{|c|c|c|c|c|}
\hline & \multicolumn{2}{|l|}{ Before } & \multicolumn{2}{|l|}{ After } \\
\hline & $201 X .4 \sim 10$ & $\begin{array}{l}\text { Mean } \\
\text { (Month) }\end{array}$ & $201 X .10 \sim 201 X+3$ & $\begin{array}{l}\text { Mean } \\
\text { (Month) }\end{array}$ \\
\hline Fall & 83 & 11.9 & 38 & 7.6 \\
\hline Bruise & 6 & 0.9 & 4 & 0.8 \\
\hline Drug & 6 & 0.9 & 5 & 1.0 \\
\hline Swallow & 0 & 0 & 1 & 0.2 \\
\hline Bed & 2 & 0.3 & 0 & 0 \\
\hline Loss & 0 & 0 & 0 & 0 \\
\hline
\end{tabular}


Clinically, we know that AD patients who manifest recent memory deficit can maintain intact remote memory, and that they can retain their skills. We considered the patients life history and designed a psychosocial intervention program that was aligned with the patients remote memories and skills. Good emotional relationships between the patients and staff, as shown by perfect participation rates, can enhance the positive effect of the intervention content.

\section{Effect of combined donepezil administration and psychosocial intervention}

The results in Analysis 2 (N2) revealed several things. The effects of donepezil on MMSE were not apparent unless the psychosocial intervention was added. This meant that the drug was considered to be ineffective according to the MMSE criteria for drug responders. This was probably due to the limitation of the dose of $10 \mathrm{mg} /$ day of the drug, and while the use of $23 \mathrm{mg} /$ day donepezil is anticipated, it is not yet permitted in Japan.

However, when the psychosocial intervention was provided in combination with the drug, the MMSE-based response ratio was calculated as $37.5 \%$. All patients receiving the combined drug and psychosocial interventions (IDs \#9 through 16) were introduced smoothly for rehabilitation and one patient (ID \#9) was discharged from N2 and returned to her home. Previous reports have indicated that the drug could stimulate attention through the frontal-parietal or basal ganglia networks [25-27]. The preservation of function of the patients, even in the severe stage of AD, was suspected to be activated by psychosocial intervention, after stimulation of the patients attention function by donepezil. The decreased rate of falling was also suspected to be due to such a combined effect. These findings also suggest that psychosocial intervention could be considered to be an outcome of the donepezil treatment.

The financial costs of combining of drug and psychosocial intervention might worry LTHCF managers. However, after an effective combining intervention, the ratio of discharge of the patients to their homes might increase like ID \#9. This increased turnover can obtain additional income by the LTCI.

\section{Limitation of the study}

In this study, we could examine only two LTCHFs. Indeed, it is not easy to involve LTCHFs for research, especially for drug treatment, since it is directly connected to the matter of management. The N1 and N2 facilities have close relationships with our laboratory, and patients there have been able to undergo CT or MRI for the purpose of research. Therefore, we should cautious about the institution bias in interpreting the results. For statistical analyses, we did not perform a three-way design
(Institute*drug*psychosocial intervention) due to the limited numbers of patients. Regarding the outcomes, we used the Vitality Index, an observational scale, which is affected by the skill of the observers. However, the Japanese version is standardized and the 3 ratings are clinically available. Also, unlikely to the Severe Impairment Battery (SIB) [28], MMSE score is not an appropriate measure for patients with severe AD. Potential cognitive changes might not have been detected, which can lead to less accurate results. Although the above-noted limitations were present, we believe the results could provide useful information on the therapeutic approach for severe AD patients in LTCHFs.

\section{Competing interests}

The authors declare that they have no competing interests.

\section{Authors contributions}

KM participated in the design of the study and MK performed the statistical analysis. YO and KA carried out the evaluation of patients in the LTHCFs. All authors read and approved the final manuscript.

Received: 28 August 2014 Accepted: 8 December 2014

Published online: 17 December 2014

\section{References}

1. Mohs RC, Doody RS, Morris JC, leni JR, Rogers SL, Perdomo CA, Pratt RD, 312 Study Group: A 1-year, placebo-controlled preservation of function survival study of donepezil in AD patients. Neurology 2001, 57:481 488.

2. Gauthier S, Lopez OL, Waldermar G, Jones RW, Cummings J, Zhang R, Schindler R, Schwam E: Effects of donepezil on activities of daily living: integrated analysis of patient data from studies in mild, moderate and severe Alzheimer s disease. Int Psychogeriatr 2010, 22:973 983.

3. Wimo A, Winblad B, Shah SN, Chin W, Zhang R, McRae T: Impact of donepezil treatment for Alzheimers disease on caregiver time. Curr Med Res Opin 2004, 20:1221 1225

4. Hebert R, Dubois MF, Wolfson C, Chambers L, Cohen C: Factors associated with long-term institutionalization of older people with dementia: data from the Canadian study of health and aging. J Gerontol A Biol Sci Med Sci 2001, 56:M693 M699.

5. Livingston G, Katona C, Francois C, Guilhaume C, Cochran J, Sapin C: Characteristics and health status change over 6 months in people with moderately severe to severe Alzheimers disease in the UK. Int Psychogeriatr 2006, 18:527 538 .

6. Geldmacher DS, Frolich L, Doody RS, Erkinjuntti T, Vellas B, Jones RW, Banerjee S, Lin P, Sano M: Realistic expectations for treatment success in Alzheimers disease. J Nutr Health Aging 2006, 10:417 429.

7. Edvardsson D, Winblad B, Sandman PO: Person-centred care of people with severe Alzheimer s disease: current status and ways forward. Lancet Neurol 2008, 7:362 367.

8. Feldman H, Gauthier S, Hecker J, Vellas B, Subbiah P, Whalen E: Donepezil MSAD Study Investigators Group: A 24-week, randomized, double-blind study of donepezil in moderate to severe Alzheimer s disease. Neurology 2001, 57:613 620 .

9. Tariot PN, Cummings $J \mathrm{~L}$, Katz IR, Mintzer J, Perdomo CA, Schwam EM, Whalen E: A randomized, double-blind, placebo-controlled study of the efficacy and safety of donepezil in patients with Alzheimer s disease in the nursing home setting. J Am Geriatr Soc 2001, 49:1590 1599.

10. Winblad B, Kilander L, Eriksson S, Minthon L, Batsman S, Wetterholm AL, Jansson-Blixt C, Haglund A, Severe Alzheimer s Disease Study Group: Donepezil in patients with severe Alzheimer s disease: double-blind, parallel-group, placebo-controlled study. Lancet 2006, 367:1057 1065.

11. Black SE, Doody R, Li H, McRae T, Jambor KM, Xu J, Sun Y, Perdomo CA, Richardson S: Donepezil preserves cognition and global function in patients with severe Alzheimers disease. Neurology 2007, 69:459 469. 
12. Jelic V, Haglund A, Kowalski J, Langworth S, WInbland B: Donepezil treatment of severe Alzheimers disease in nursing home settings: A responder analysis. Dement Geriatr Cogn Disord 2008, 26:458 466.

13. Grasel E, Wiltfang J, Kornhuber J: Non-drug therapies for dementia: an overview of the current situation with regard to proof of effectiveness. Dement Geriatr Cogn Disord 2003, 15:115 125.

14. Zec RF: Neuropsychological Functioning in Alzheimers Disease. In Neuropsychol Alzheimers Disease and Other Dementias. Edited by Parks RW, Zec RF, Wilson RS. Oxford: Oxford University Press; 1993:3 80.

15. Onder G, Zanetti O, Giacobini E, Frisoni GB, Bartorelli L, Carbone G, Lambertucci P, Silveri MC, Bernabei R: Reality orientation therapy combined with cholinesterase inhibitors in Alzheimer s disease: randomized controlled trial. Br J Psychiatr 2005, 187:450 455

16. Spector A, Orrell M, Davies S, Woods B: Reality orientation for dementia. Cochrane Database Syst Rev 2007, (3):CD001119.

17. Woods B, Spector A, Jones C, Orrell M, Davies S: Reality orientation therapy for dementia. Cochrane Database Syst Rev 2005, (2):CD001120.

18. Meguro M, Kasai M, Akanuma K, Ishii H, Yamaguchi S, Meguro K: Comprehensive approach of donepezil and psychosocial intervention on cognitive function and quality of life for Alzheimers disease: the Osaki-Tajiri project. Age Ageing 2008, 37:469 473.

19. Meguro K: Preventing withdrawal of cholinesterase inhibitors for Alzheimers patients: measures from three aspects. Psychogeriatrics 2014 [Epub].

20. Umegaki H, Itoh A, Suzuki Y, Nabeshima T: Discontinuation of donepezil for the treatment of Alzheimers disease in geriatric practice. Int Psychogeriatr 2008, 20(4):800 806

21. Giordano M, Dominguez $\amalg$, Vitrano T, Curatolo M, Ferlisi A, Prima AD, Belvedere M, Barbagallo M: Combination of intensive cognitive rehabilitation and donepezil therapy in Alzheimer s disease (AD). Arch Gerontol Geriatr 2010, 52:245 249.

22. McKhann G, Drachman D, Folstein M, Katzman R, Price D, Stadlan EM: Clinical diagnosis of Alzheimers disease: report of the NINCDS-ADRDA work group under the auspices of department of health and human services task force on Alzheimers disease. Neurology 1984, 34:939 944

23. Folstein MF, Folstein SE, McHugh PR: Mini-mental state : a practical method for grading the cognitive state of patients for the clinician. J Psychiatr Res 1975, 12:189 198.

24. Toba K, Nakai R, Akishita M, lijima S, Nishinaga M, Mizoguchi T: Vitality index as a useful tool to assess elderly with dementia. Geriatr Gerontol Int 2002, 2:23 29.

25. Nobili F, Koulibaly M, Vitali P, Migneco O, Mariani G, Ebmeier K, Pupi A, Robert PH, Rodriguez G, Darcourt J: Brain perfusion follow-up in Alzheimers patients during treatment with acetylcholinesterase inhibitors. J Nucl Med 2002, 43:983 990.

26. Nakano S, Asada T, Matsuda H, Uno M, Takasaki M: Donepezil hydrochloride preserves regional cerebral blood flow in patients with Alzheimers disease. J Nucl Med 2001, 42:1441 1445.

27. Tune L, Tiseo PJ, leni J, Perdomo C, Pratt RD, Votaw JR, Jewart RD, Hoffman JM: Donepezil $\mathrm{HCl}$ (E2020) maintains functional brain activity in patients with Alzheimer disease: results of a 24-week, double-blind, placebo-controlled study. Am J Geriatr Psychiatr 2003, 11(2):169 177.

28. Panisset M, Roudier M, Saxton J, Boller F: Severe impairment battery: a neuropsychological test for severely demented patients. Arch Neurol 1994, 51:41 45.

\section{doi:10.1186/s12883-014-0243-7}

Cite this article as: Meguro et al:: Donepezil can improve daily activities and promote rehabilitation for severe Alzheimers patients in long-term care health facilities. BMC Neurology 2014 14:243. 